\title{
Transarterial chemoembolization (TACE) combined with apatinib versus TACE combined with sorafenib in advanced hepatocellular carcinoma patients: a multicenter retrospective study
}

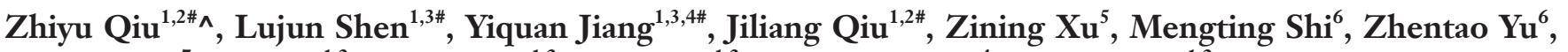 \\ Yanping $\mathrm{Ma}^{7}$, Wei He ${ }^{1,2}$, Yun Zheng ${ }^{1,2}$, Binkui Li ${ }^{1,2}$, Guoying Wang ${ }^{4}$, Yunfei Yuan ${ }^{1,2} \wedge$ \\ ${ }^{1}$ State Key Laboratory of Oncology in South China and Collaborative Innovation Center of Cancer Medicine, Sun Yat-sen University, Guangzhou, \\ China; ${ }^{2}$ Department of Liver Surgery, Sun Yat-sen University Cancer Center, Guangzhou, China; ${ }^{3}$ Department of Minimally Invasive Interventional \\ Therapy, Sun Yat-sen University Cancer Center, Guangzhou, China; ${ }^{4}$ Department of Hepatic Surgery and Liver Transplantation Center, the Third \\ Affiliated Hospital of Sun Yat-sen University, Guangzhou, China; ${ }^{5}$ Department of Minimally Invasive Interventional Radiology, the Second Affiliated \\ Hospital of Guangzhou Medical University, Guangzhou, China; ${ }^{6}$ Zhongshan School of Medicine, Sun Yat-sen University, Guangzhou, China; \\ ${ }^{7}$ Department of Radiology, the Third Affiliated Hospital of Sun Yat-sen University, Guangzhou, China \\ Contributions: (I) Conception and design: Y Yuan, G Wang, Z Qiu; (II) Administrative support: Y Yuan, G Wang, L Shen, Y Jiang, J Qiu; (III) \\ Provision of study materials or patients: Y Yuan, G Wang, B Li, Y Zheng, J Qiu, W He, Z Xu; (IV) Collection and assembly of data: Z Qiu, Z Xu, M \\ Shi, Z Yu, Y Ma; (V) Data analysis and interpretation: Z Qiu, J Qiu, W He, Y Zheng, B Li; (VI) Manuscript writing: All authors; (VII) Final approval \\ of manuscript: All authors. \\ "These authors contributed equally to this work. \\ Correspondence to: Yunfei Yuan, MD. State Key Laboratory of Oncology in South China and Collaborative Innovation Center of Cancer Medicine, \\ Sun Yat-sen University, No. 651 Dongfeng East Road, Guangzhou 510060, China. Email: yuanyf@mail.sysu.edu.cn; Guoying Wang, MD, PhD. \\ Department of Hepatic Surgery and Liver Transplantation Center, the Third Affiliated Hospital of Sun Yat-sen University, No. 600 Tianhe Road, \\ Guangzhou 510630, China. Email: wanggy3@126.com.
}

Background: The combination of transarterial chemoembolization (TACE) with sorafenib has demonstrated superior efficacy over sorafenib and TACE monotherapy in hepatocellular carcinoma (HCC). Apatinib, a new targeted agent, has been recently reported to prolong the survival of HCC patients, either alone or in combination with TACE. However, the superior regimen between TACE-apatinib and TACEsorafenib in HCC patients has not been determined. In this study, we compared the efficacy and safety of TACE-apatinib versus TACE-sorafenib in advanced stage HCC patients.

Methods: The data of 201 HCC patients who had received TACE-sorafenib or TACE-apatinib between January 2016 and June 2018 in three hospitals were retrospectively reviewed. Overall survival (OS), progression-free survival (PFS), and adverse effects (AEs) between the two treatment groups were compared. A subgroup analysis based on the doses of targeted agents was also performed.

Results: No significant differences in baseline clinicopathological features were found between the two groups except for dose reduction. The TACE-apatinib group had higher incidences of hypertension, oral or anal ulcer and proteinuria, while the TACE-sorafenib group had higher incidences of diarrhea and alopecia. Grade 3/4 AEs occurred more frequently in the TACE-apatinib group than in the TACE-sorafenib group (52.3\% vs. 22.6\%, $\mathrm{P}<0.001$ ). The TACE-sorafenib group had better PFS than the TACE-apatinib group (median PFS: 5.0 vs. 6.0 months, $\mathrm{P}=0.002$ ) while the two groups showed no difference in OS (median OS: 13.0 vs. 13.0 months, $\mathrm{P}=0.448$ ). The TACE-apatinib group had a higher rate of targeted agent dose reduction than the TACE-sorafenib group $(53.5 \%$ vs. $17.4 \%, \mathrm{P}<0.001)$. When the patients were stratified into normal and reduced-dose subgroups, those who received TACE-sorafenib exhibited improved PFS but similar OS compared with the patients who received TACE-apatinib in the reduced-dose subgroup (median OS: 12.0

^ ORCID: Zhiyu Qiu, 0000-0001-6725-6439; Yunfei Yuan, 0000-0003-2467-3683. 
vs. 13.3 months, $\mathrm{P}=0.614$; median $\mathrm{PFS}: 3.0$ vs. 7.0 months, $\mathrm{P}<0.001)$. Multivariable analysis validated that treatments and dose reduction were independent prognostic factors for PFS among all patients.

Conclusions: Compared with TACE-sorafenib, the strategy of TACE-apatinib yielded shorter PFS in advanced HCC patients while no difference in OS was observed. A high rate of AE-related dose reduction of apatinib could account for the observed differences.

Keywords: Hepatocellular carcinoma (HCC); transarterial chemoembolization (TACE); sorafenib; apatinib; prognosis

Submitted Jul 17, 2020. Accepted for publication Nov 20, 2020.

doi: $10.21037 /$ atm-20-5360

View this article at: http://dx.doi.org/10.21037/atm-20-5360

\section{Introduction}

In the past decade, the incidence of hepatocellular carcinoma (HCC) has increased despite a downward trend in the incidence of all tumors, and the East Asian region accounts for a substantial portion of new cases $(1,2)$. More than $60 \%$ of HCC cases are diagnosed at an intermediate or advanced stage, mostly not amenable for curative therapies (3). Based on the clinical guideline of the European Association for the Study of the Liver, systemic therapy is the current recommended treatment for advanced HCC [Barcelona Clinic Liver Cancer (BCLC) stage C] (4). Sorafenib, a multi-kinase inhibitor that blocks tumor angiogenesis and growth, is recognized as the first targeted agent for advanced HCC. However, sorafenib has demonstrated modest efficacy by prolonging the median overall survival (OS) of HCC patients by 2.8 months compared with placebo (5). In the Asia-Pacific region, this survival benefit was even more marginal (OS: 6.5 vs. 4.2 months) (6). Subsequently, a combination of transarterial chemoembolization (TACE) with sorafenib is recommended to exert a synergistic therapeutic effect in unresectable HCC. The underlying rationale is that sorafenib could inhibit neo-angiogenesis after TACE $(7,8)$. Several trials have demonstrated the feasibility and superior efficacy of this combination therapy in HCC patients compared to sorafenib or TACE monotherapy (9-11). However, unsatisfactory response rate and heterogeneous survival benefits still motivate researchers to seek for new targeted agents, improved interventional therapies and optimal combinations (12-16).

Apatinib is a novel tyrosine kinase inhibitor (TKI) with strong selectivity to vascular endothelial growth factor receptor (VEGFR)-2 (17). In 2014, a phase II clinical study reported that apatinib monotherapy could prolong the OS of advanced HCC patients (18). Considering its potential inhibitory role in post-TACE neo-angiogenesis, a series of studies have shown clinical benefits of TACE-apatinib in unresectable HCC compared with TACE-alone (19-24). However, no previous comparison between TACE-apatinib and TACE-sorafenib has been conducted.

We herein compared the efficacy and safety of TACEapatinib treatment versus TACE-sorafenib treatment in advanced HCC patients. We present the following article in accordance with the STROBE reporting checklist (available at http://dx.doi.org/10.21037/atm-20-5360).

\section{Methods}

\section{Study design}

This retrospective study included patients from three academic hospitals in China (the Sun Yat-sen University Cancer Center, the Third Affiliated Hospital of Sun Yatsen University, and the Second Affiliated Hospital of Guangzhou Medical University) between January 2016 and June 2018. This study was approved by the ethics committees of all three hospitals (No. B2019-153) in accordance with the ethical principles of the Declaration of Helsinki (as revised in 2013). Written informed consent was not required due to the retrospective nature of this study.

Inclusion criteria for this study were the following: (I) age between 18-75 years; (II) stage $\mathrm{C}$ according to the BCLC staging system; (III) Child-Pugh (CP) Class A or $\mathrm{B}$ liver function; and (IV) a score of 0 or 1 according to the Eastern Cooperative Group performance status. The exclusion criteria were as follows: (I) heart, lung, or kidney dysfunction; (II) inadequate bone marrow function (white blood cell count $<3.0 \times 10^{9} / \mathrm{L}$, platelet count $<40 \times 10^{9} / \mathrm{L}$, or absolute neutrophil count $<1.5 \times 10^{9} / \mathrm{L}$ ); (III) other primary 
malignancies or immune deficiencies; (IV) concomitant antineoplastic treatments except TACE, sorafenib, or apatinib before disease progression in this study; (V) concomitant administration of sorafenib and apatinib or switching to the opposite treatment before disease progression in this study; and (VI) an interval over 15 days between TACE and the administration of targeted agents. All patients in this study had been informed of choices of targeted agents and they made their decisions based on the full communication between patients and doctors as well as their personal willingness. Accordingly, the patients were classified into two groups, namely the TACE-sorafenib and the TACE-apatinib group.

\section{Therapeutic method}

\section{TACE}

TACE was performed by experienced radiologists in the three hospitals using similar protocols. After intubation, the angiography of hepatic and related vessels was performed to evaluate the blood supply to the liver and the feasibility of TACE. Once the catheterization of tumor-feeding arteries was completed, chemotherapeutic agents were mixed and infused via a catheter inserted into the arteries. The infusion emulsion contained 30-50 mg of epirubicin (Pharmorubicin; Pfizer, Jiangsu, China), 30-50 mg of lobaplatin (Hainan Changan International Pharmaceutical Co., Ltd., Hainan, China), and 5-15 mL of lipiodol (Lipiodol Ultrafluide; France). Absorbable gelatin sponge particles (Gelfoam; Hangzhou alc Ltd., Zhejiang, China) were subsequently used to embolize the arteries. Response to TACE was evaluated 1 month after the operation via contrast-enhanced computed tomography (CT) or magnetic resonance imaging (MRI). Repeated TACE was recommended if shrinkage of lipiodol deposition, recurrence of residual lesions, or intrahepatic progression was observed on follow-up imaging, using a similar treatment protocol as the initial operation.

\section{Administration of targeted agents}

The administration was performed within 15 days after first TACE operation and continued until intolerable adverse events (AEs) occurred or disease progression developed. Initially, $500 \mathrm{mg}$ of daily apatinib (Hengrui Pharmaceutical Co., Ltd., Shanghai, China) and $400 \mathrm{mg}$ of sorafenib (Bayer Health-care, Leverkusen, Germany) twice daily were prescribed, respectively. If the patients could not tolerate the initial dose, stepwise dose modification was recommended as follows: from $250 \mathrm{mg}$ daily to $250 \mathrm{mg}$ every other day for apatinib, and from $600 \mathrm{mg}$ daily to $400 \mathrm{mg}$ daily, then to $400 \mathrm{mg}$ every other day for sorafenib $(21,25)$. Re-escalation to the last-step dose was advised once the AEs were alleviated or the patients could tolerate them.

\section{Follow-up, endpoints, and evaluation of therapeutic effect}

The patients were regularly followed up and examined using abdominal contrast-enhanced CT or MRI every 4-6 weeks during follow-up periods. OS and progression-free survival (PFS) were compared between the two groups. OS was defined as the interval between the date of first treatment and the date of death or last follow-up (October 1, 2019). PFS was defined as the interval between the date of first treatment and the date of disease progression, death, or last follow-up. According to the modified response evaluation criteria in solid tumor, the patients' imaging examination results were evaluated by at least two radiologists with 10 years of experience, using the following 4 levels of treatment responses: complete response (CR), partial response (PR), stable disease (SD), and progressive disease (PD) (26). Referring to a previous study, disease control rate (DCR) was defined as the proportion of patients who maintained a tumor response of CR, PR, or SD for at least 12 weeks from first manifestation (27). AEs were recorded and graded according to the Common Terminology Criteria for Adverse Events (version 4.0).

\section{Statistics analysis}

Analyses were performed using SPSS 24.0 (SPSS Inc., Chicago, IL, USA) and R 3.6.0 software (R Foundation for Statistical Computing, Vienna, Austria). Unless indicated otherwise, comparison was conducted using the chi-square test for categorical variables and the $t$-test for continuous variables. OS and PFS were estimated using the Kaplan-Meier method and compared using the log-rank test. The prognostic value of potential predictors was assessed using the Cox proportional hazard regression model. Only variables with $\mathrm{P}$ value $<0.10$ in univariable analysis were added in multivariable analysis for identifying independent prognostics factors affecting OS or PFS. Inspection level was set at $\alpha=0.05$.

\section{Results}

\section{Baseline characteristics}

Between January 2016 and June 2018, a total of 201 HCC 


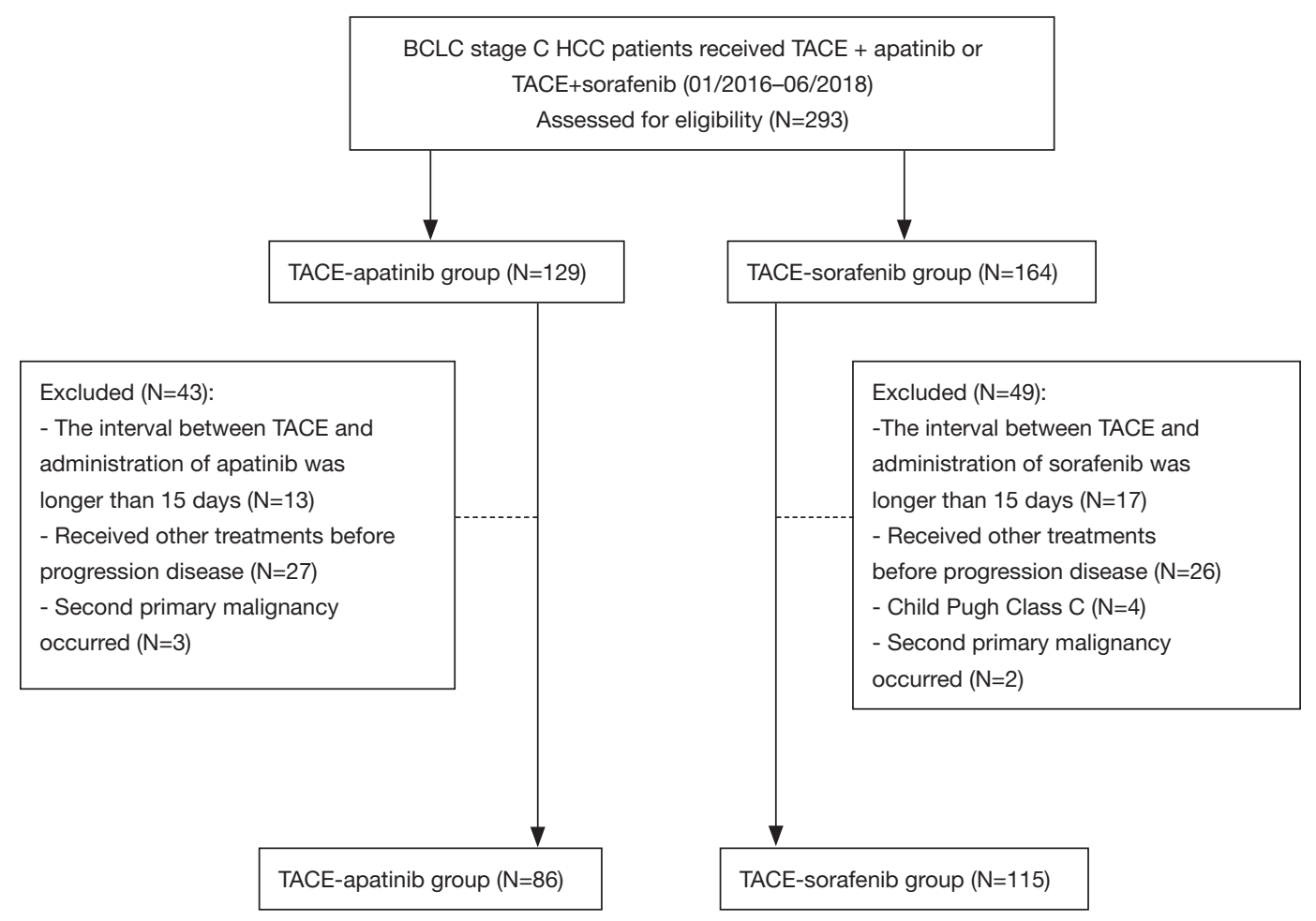

Figure 1 Flow chart. BCLC Barcelona Clinic Liver Cancer, HCC, hepatocellular carcinoma; TACE, transarterial chemoembolization.

patients who had undergone TACE-apatinib $(n=86)$ or TACE-sorafenib $(n=115)$ treatment in the three hospitals were evaluated (Figure 1). The median age of these patients was 52 (range, 23-75) years. The baseline characteristics of the two groups are listed and compared in Table 1. All patients were diagnosed with BCLC stage C HCC and 116 of them had distant metastasis while 126 had major vascular invasion. Differences in the characteristics were not significant between the two groups except for dose reduction. Particularly, the rate of dose reduction was higher in the TACE-apatinib group than in the TACEsorafenib group $(53.5 \%$ vs. $17.4 \%, \mathrm{P}<0.001)$.

\section{AEs and safety assessment}

Treatment-related AEs are listed in Table 2. Hand-foot syndrome was the most common AE with similar incidences in both groups (TACE-apatinib: 58.1\%; TACE-sorafenib: $51.3 \% ; \mathrm{P}=0.336)$. Other common AEs in the TACE-apatinib group were hypertension (39.5\%), proteinuria (37.2\%), and oral or anal ulcer $(32.6 \%)$. In the TACE-sorafenib group, patients also suffered from diarrhea (31.3\%), fever (30.4\%), and abdominal pain $(26.1 \%)$. In comparison, the TACE- apatinib group had higher incidences of hypertension, oral or anal ulcer and proteinuria while the TACE-sorafenib group had higher incidences of diarrhea and alopecia (all $\mathrm{P}<0.05)$. These AEs were predominantly related to the targeted agents, while TACE-related AEs such as fever, abdominal pain, and pleural effusion were comparable in the two groups. Moreover, the TACE-apatinib group had a higher incidence of severe (grade 3/4) AEs than the TACEsorafenib group $(52.3 \%$ vs. $22.6 \%, \mathrm{P}<0.001)$. Only one patient developed TACE-related severe AEs, but the TACE protocol was not affected.

Overall, 66 of the 201 patients had permanently reduced the doses or discontinued the drug administration due to the AEs before disease progression (TACE-apatinib, $\mathrm{n}=46$; TACE-sorafenib, $\mathrm{n}=20$ ). After dose reduction and symptomatic treatments, their symptoms gradually receded. The rest of the patients developed tolerable AEs and eventually resumed the initial dose. No hepatic failure, unexpected AEs, or grade $5 \mathrm{AEs}$ were observed.

\section{Survival analysis}

During the follow-up, 134 of the 201 patients died from 
Table 1 Baseline characteristics

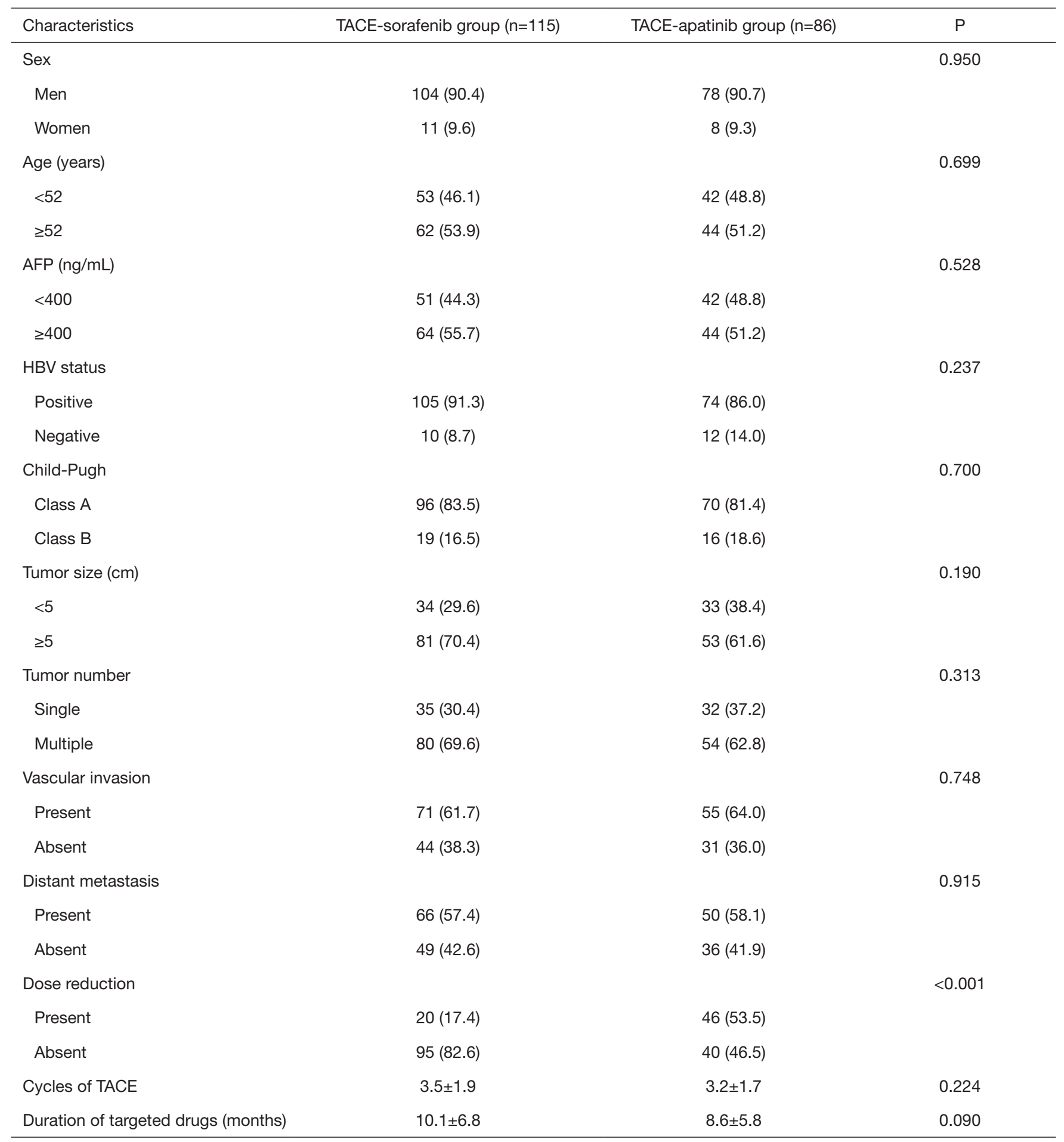

Data are shown as numbers of events with percentages in parentheses or averages \pm standard deviations. TACE, transarterial chemoembolization; AFP, alpha fetoprotein; HBV, hepatitis B virus. 
Table 2 Treatment-emergent adverse events in two groups

\begin{tabular}{|c|c|c|c|c|c|c|c|c|c|}
\hline Adverse events & \multicolumn{3}{|c|}{ TACE-sorafenib group $(n=115)$} & \multicolumn{3}{|c|}{ TACE-apatinib group $(n=86)$} & \multicolumn{3}{|c|}{$\mathrm{P}$} \\
\hline All & $115(100.0)$ & $89(77.4)$ & $26(22.6)$ & $86(100.0)$ & $41(47.7)$ & $45(52.3)$ & 1.000 & $<0.001$ & $<0.001$ \\
\hline Hand-foot syndrome & $59(51.3)$ & $42(36.5)$ & $17(14.8)$ & $50(58.1)$ & $23(26.7)$ & $27(31.4)$ & 0.336 & 0.143 & 0.005 \\
\hline Fever & $35(30.4)$ & $35(30.4)$ & $0(0.0)$ & $27(31.4)$ & $27(31.4)$ & $0(0.0)$ & 0.884 & 0.884 & - \\
\hline Fatigue & $17(14.8)$ & $15(13.0)$ & $2(1.7)$ & $17(19.8)$ & $14(16.3)$ & $3(3.5)$ & 0.351 & 0.518 & 0.741 \\
\hline Hypertension & $26(22.6)$ & $24(20.9)$ & $2(1.7)$ & $34(39.5)$ & $26(30.2)$ & $8(9.3)$ & 0.009 & 0.129 & 0.035 \\
\hline Nausea/vomiting & $20(17.4)$ & $18(15.7)$ & $2(1.7)$ & $13(15.1)$ & $8(9.3)$ & $5(5.8)$ & 0.667 & 0.184 & 0.242 \\
\hline Anorexia & $12(10.4)$ & $10(8.7)$ & $2(1.7)$ & $9(10.5)$ & $3(3.5)$ & $6(7.0)$ & 0.994 & 0.138 & 0.130 \\
\hline Oral or anal ulcer & $23(20.0)$ & $21(18.3)$ & $2(1.7)$ & $28(32.6)$ & $13(15.1)$ & $15(17.4)$ & 0.043 & 0.556 & $<0.001$ \\
\hline Proteinuria & $9(7.8)$ & $8(7.0)$ & $1(0.9)$ & $32(37.2)$ & $22(25.6)$ & $10(11.6)$ & $<0.001$ & $<0.001$ & 0.003 \\
\hline Skin rash & $9(7.8)$ & $9(7.8)$ & $0(0.0)$ & $10(11.6)$ & $9(10.5)$ & $1(1.2)$ & 0.362 & 0.517 & 0.884 \\
\hline Alopecia & $26(22.6)$ & $26(22.6)$ & $0(0.0)$ & $3(3.5)$ & $3(3.5)$ & $0(0.0)$ & $<0.001$ & $<0.001$ & - \\
\hline
\end{tabular}

Data are shown as numbers of events with percentages in parentheses. TACE, transarterial chemoembolization.

tumor progression. No significant difference was observed in OS between the two groups (median OS: $13.0 \mathrm{vs}$. 13.0 months, $\mathrm{P}=0.448$ ). However, the TACE-sorafenib group exhibited improved PFS compared with the TACEapatinib group (median PFS: 5.0 vs. 6.0 months, $\mathrm{P}=0.002$ ) (Figure 2). In the TACE-apatinib group, 3 patients had PR $(3.5 \%)$ and 47 patients had SD $(54.7 \%)$. In the TACEsorafenib group, 2 patients had CR (1.7\%), 30 patients had PR (26.1\%), and 60 patients had SD (52.2\%). The DCR was $58.1 \%$ and $80.0 \%$ in the TACE-apatinib group and the TACE-sorafenib group, respectively $(\mathrm{P}=0.001)$.

Considering the higher rate of dose reduction in the TACE-apatinib group, a subgroup analysis based on the agent doses was subsequently conducted and the patients were stratified into a reduced-dose subgroup or a normaldose subgroup. In the reduced-dose subgroup, the patients had permanently reduced the doses or discontinued the administration. Their median average daily doses were $312 \mathrm{mg}$ of apatinib or $595 \mathrm{mg}$ of sorafenib. In the normaldose subgroup, the patients basically maintained the initial treatment dose and their median average daily doses were $432 \mathrm{mg}$ of apatinib or $770 \mathrm{mg}$ of sorafenib. The average daily dose was determined within the patient-based actual days of administration excluding treatment interruption. In the reduced-dose subgroup, TACE-sorafenib conferred a survival benefit in terms of PFS compared with TACEapatinib but failed in terms of OS (median OS: $12.0 \mathrm{vs}$. 13.3 months, $\mathrm{P}=0.614$; median PFS: 3.0 vs. 7.0 months, $\mathrm{P}<0.001)$. In the normal-dose subgroup, no significant differences in terms of OS and PFS were observed between the two treatment arms (median OS: 14.0 vs. 13.0 months, $\mathrm{P}=0.710$; median PFS: 7.0 vs. 6.0 months, $\mathrm{P}=0.918$ ), respectively (Figure 3). Furthermore, the normal-dose subgroup had superior PFS but similar OS compared with the reduced-dose subgroup in the TACE-apatinib group (median OS: 14.0 vs. 12.0 months, $\mathrm{P}=0.827$; median PFS: 7.0 vs. 3.0 months, $\mathrm{P}<0.001)$. However, in the TACE-sorafenib group, the normal-dose subgroup had no survival benefits in terms of both OS and PFS, compared with the reduceddose subgroup (median OS: 13.0 vs. 13.3 months, $\mathrm{P}=0.788$; 

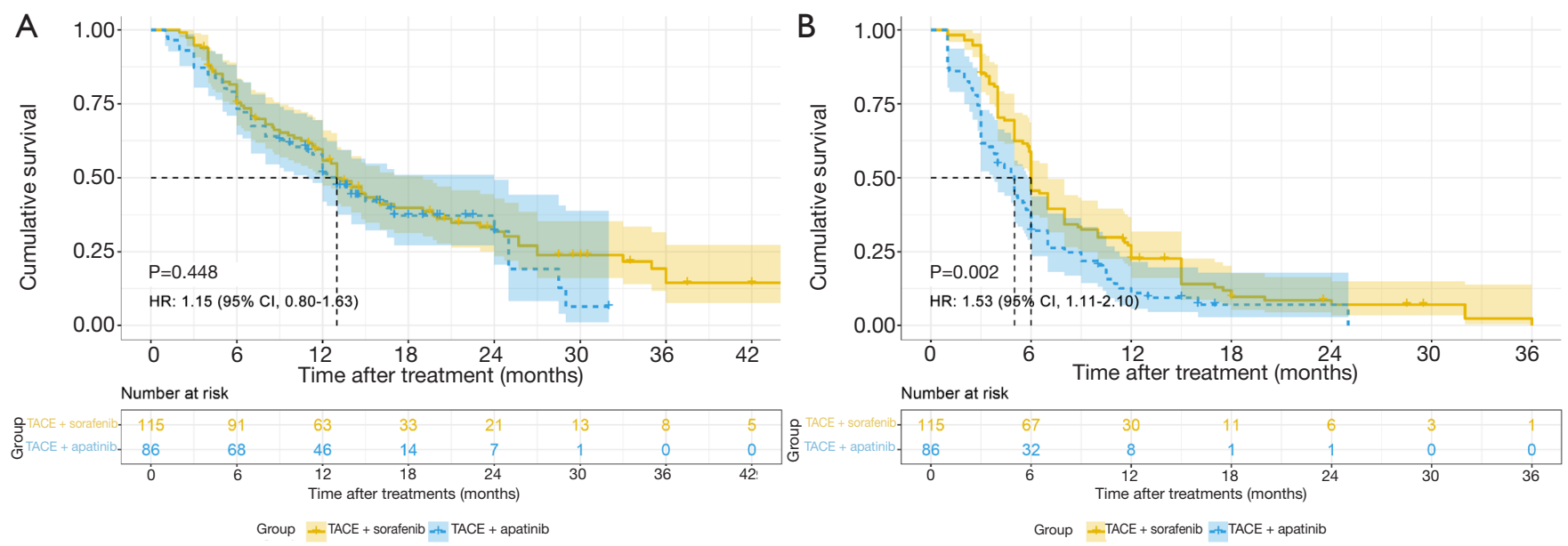

Figure 2 Kaplan-Meier survival curves. OS (A) and PFS (B) among all patients. HR, hazard ratio; CI, confidence interval; TACE, transarterial chemoembolization; OS, overall survival; PFS, progression-free survival.
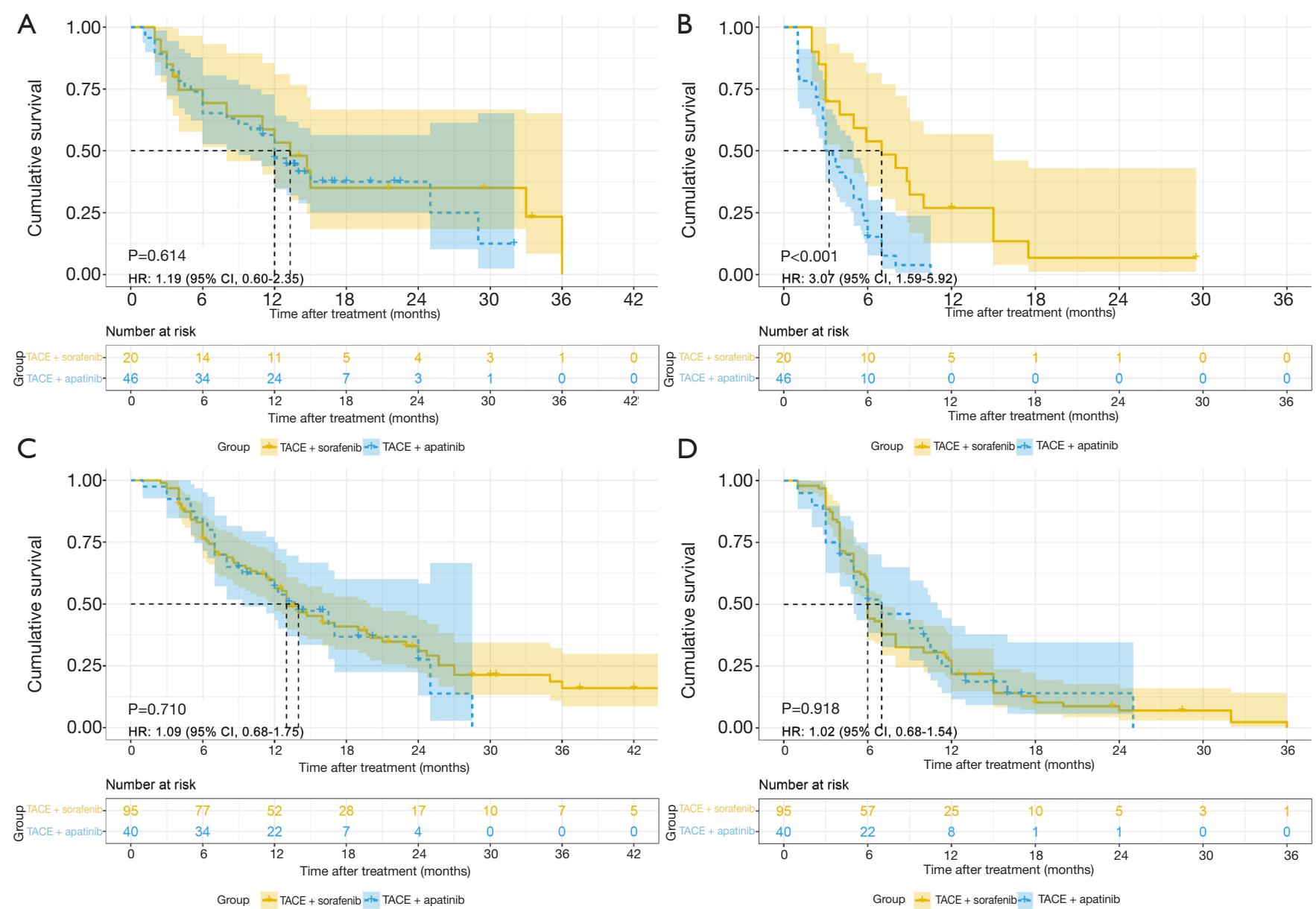

Figure 3 Kaplan-Meier survival curves. (A) OS in the reduced-dose subgroup; (B) PFS in the reduced-dose subgroup; (C) OS in the normaldose subgroup; (D) PFS in the normal-dose subgroup. HR, hazard ratio; CI, confidence interval; TACE, transarterial chemoembolization; OS, overall survival; PFS, progression-free survival. 

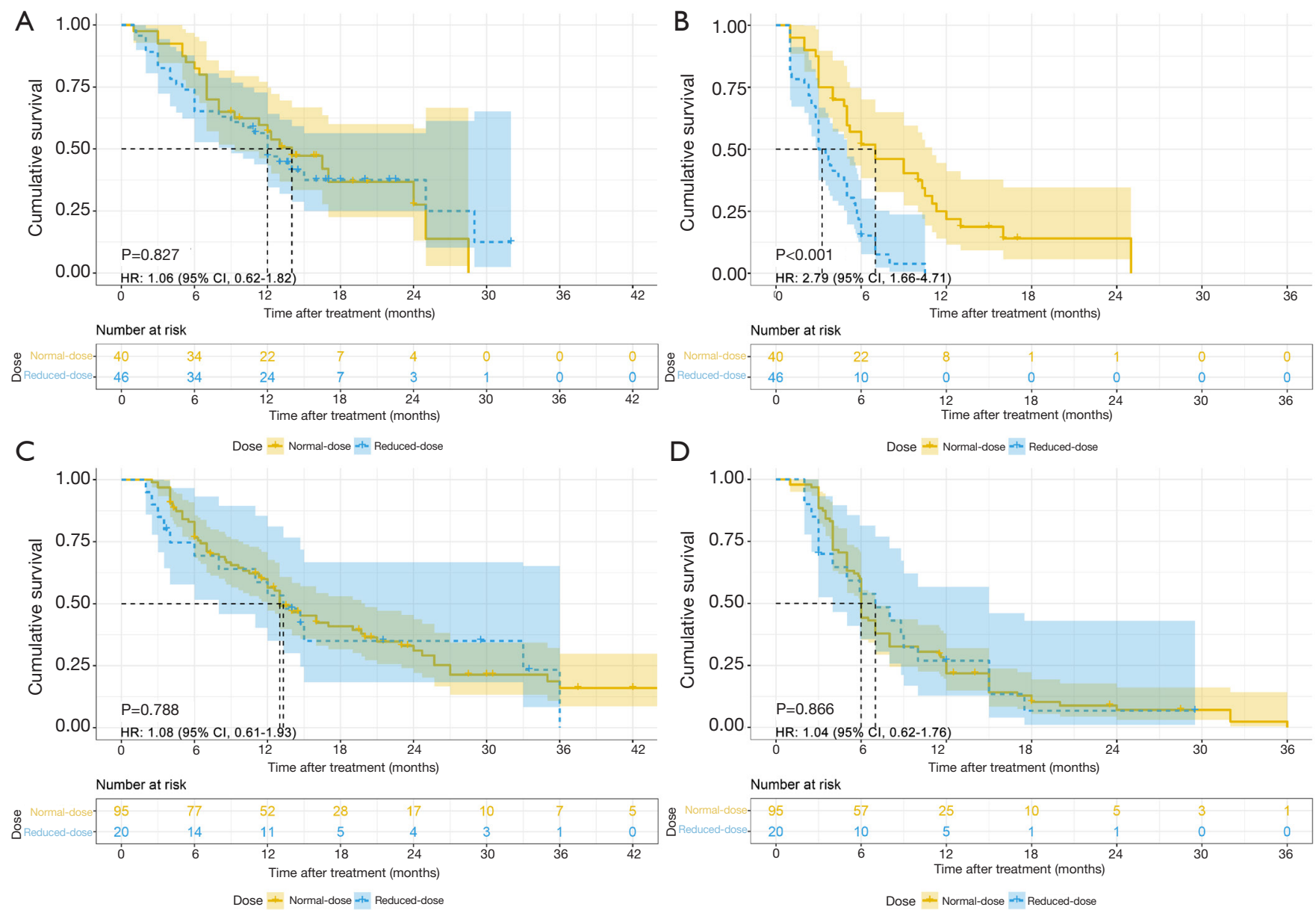

Figure 4 Kaplan-Meier survival curves. (A) OS in the TACE-apatinib group; (B) PFS in the TACE-apatinib group; (C) OS in the TACEsorafenib group; (D) PFS in the TACE-sorafenib group. HR, hazard ratio; CI, confidence interval; OS, overall survival; TACE, transarterial chemoembolization; PFS, progression-free survival.

median PFS: 6.0 vs. 7.0 months, $\mathrm{P}=0.866$ ) (Figure 4).

\section{Prognostic factors affecting OS and PFS}

Multivariable analyses confirmed that age $\geq 52$ [hazard ratio (HR): 0.57 ; $95 \%$ confidence interval (CI), 0.40-0.80; $\mathrm{P}=0.001$ ], CP class B (HR: $1.65 ; 95 \% \mathrm{CI}, 1.09-2.51$; $\mathrm{P}=0.019$ ), and tumor size $\geq 5 \mathrm{~cm}$ (HR: $1.61 ; 95 \% \mathrm{CI}, 1.10$ $2.35 ; \mathrm{P}=0.014)$ were independent prognostic factors for OS among all patients. In the reduced-dose subgroup, age $\geq 52$ (HR: $0.45 ; 95 \%$ CI, 0.23-0.87, $\mathrm{P}=0.018$ ) and $\mathrm{CP}$ class B (HR: 2.40; 95\% CI, 1.22-4.69; $\mathrm{P}=0.011$ ) were independent prognostic factors for OS. In the normal-dose subgroup, age $\geq 52$ (HR: $0.58 ; 95 \%$ CI, $0.38-0.88, \mathrm{P}=0.010$ ) was the only independent prognostic factor for OS (Table 3).

For PFS, multivariable analyses confirmed that age $\geq 52$ (HR: 0.68; 95\% CI, 0.51-0.92; $\mathrm{P}=0.012$ ), tumor size $\geq 5$ cm (HR: $1.44 ; 95 \%$ CI, 1.04-1.99; $\mathrm{P}=0.026$ ), TACEapatinib (HR: 1.53; 95\% CI, 1.11-2.10; $\mathrm{P}=0.010$ ), and dose reduction (HR: 1.65; 95\% CI, 1.19-2.28; $\mathrm{P}=0.003$ ) were independent prognostic factors for PFS among all patients. In the reduced-dose subgroup, CP class B (HR: 2.82; 95\% CI, 1.49-5.33; $\mathrm{P}=0.001$ ) and TACE-apatinib (HR: 3.07; 95\% CI, 1.59-5.92, $\mathrm{P}=0.001$ ) were independent prognostic factors for PFS. In the normal-dose subgroup, tumor size $\geq 5 \mathrm{~cm}$ (HR: $1.48 ; 95 \%$ CI, $1.01-2.17 ; \mathrm{P}=0.044$ ) was the only independent prognostic factor for PFS (Table 4).

\section{Treatments after tumor progression}

After tumor progression had been confirmed by radiological results, 83 of the 201 patients received subsequent 


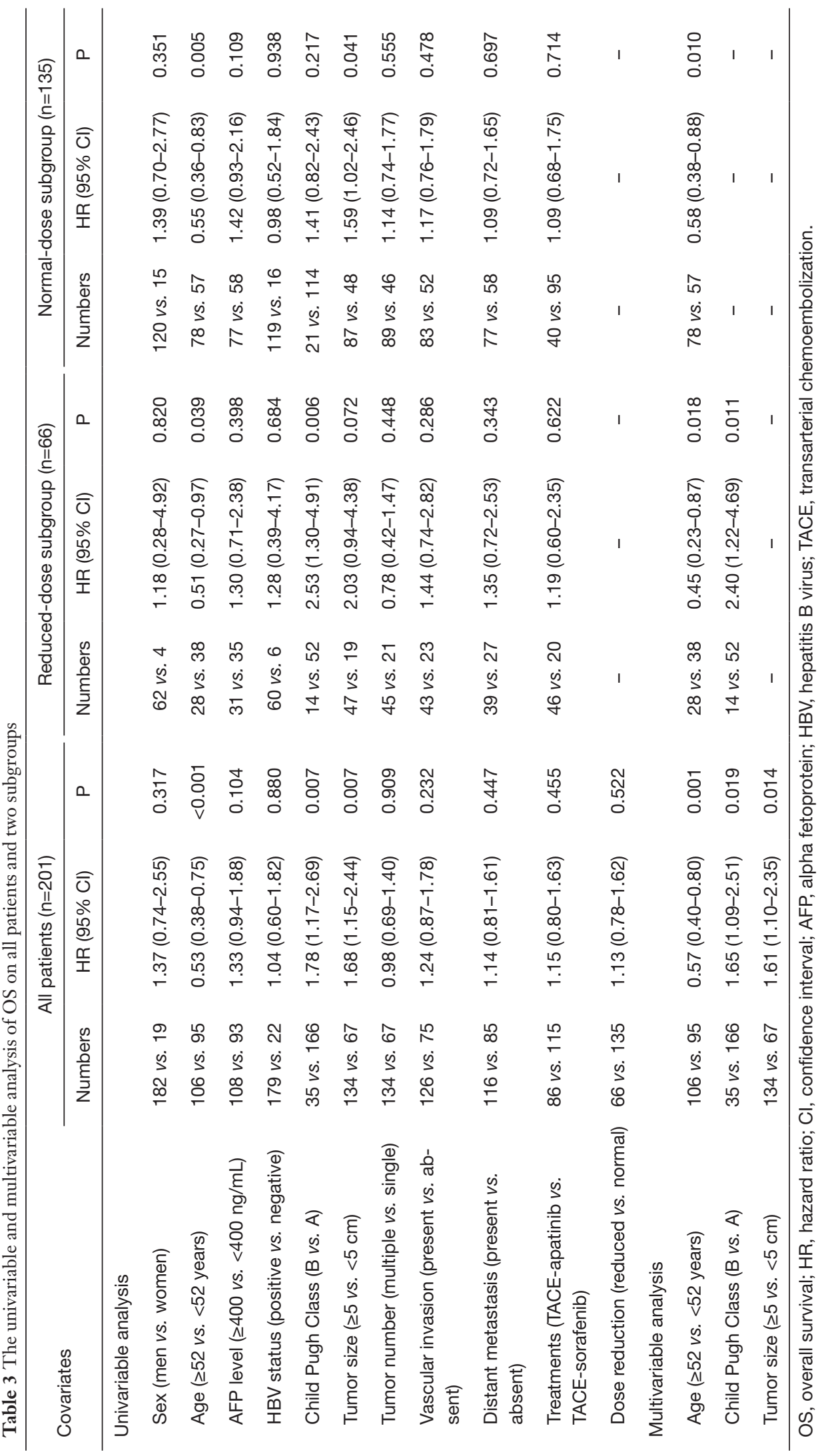




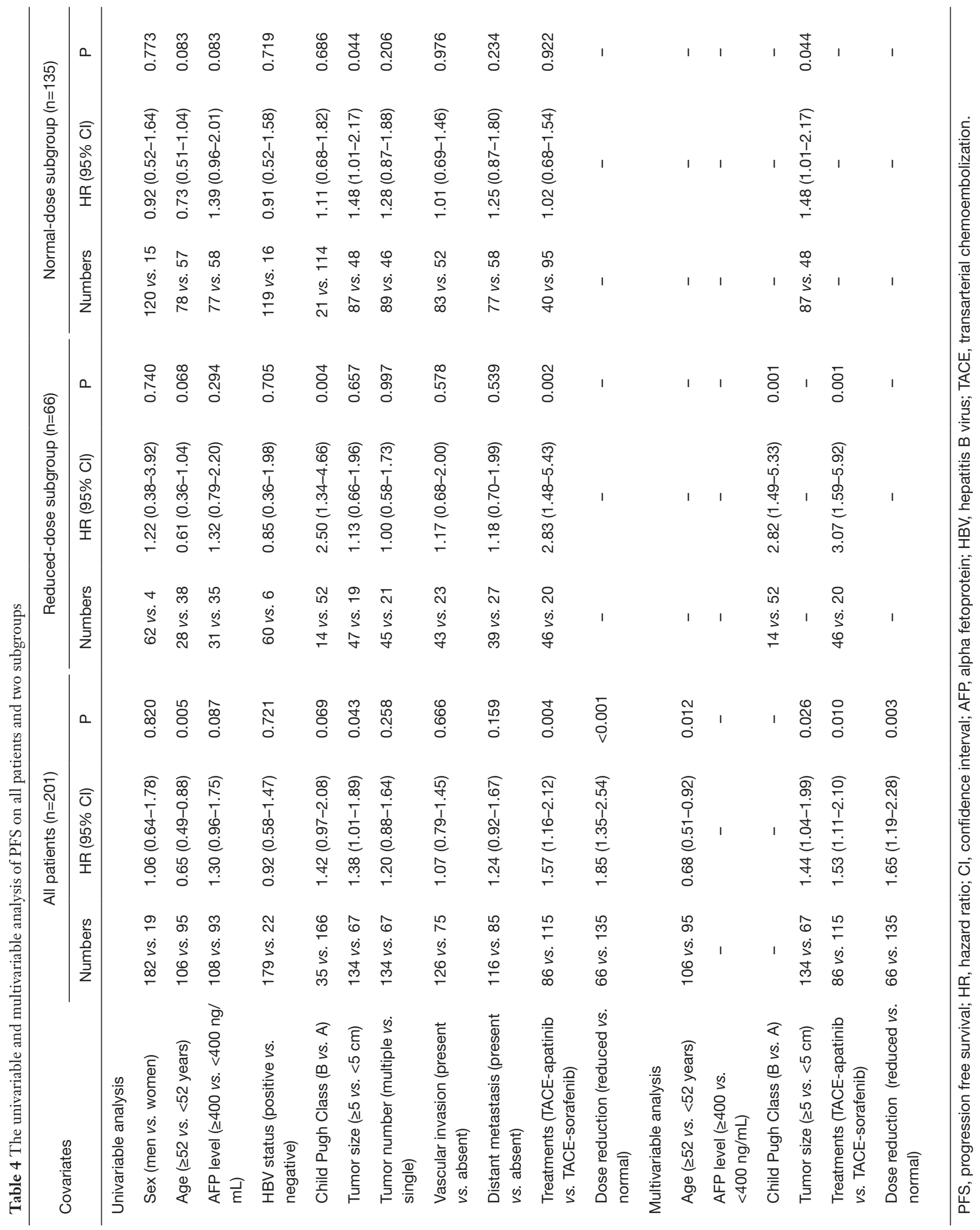


Table 5 Post-treatments after tumor progression in two groups

\begin{tabular}{|c|c|c|c|}
\hline Treatments & TACE-sorafenib group $(n=115)$ & TACE-apatinib group $(n=86)$ & $\mathrm{P}$ \\
\hline Palliative surgery & $5(4.3)$ & $2(2.3)$ & 0.700 \\
\hline $\begin{array}{l}\text { Radioactive seed implanting } \\
\text { (iodine-125) }\end{array}$ & $15(13.0)$ & $18(20.9)$ & 0.135 \\
\hline Sorafenib & $0(0.0)$ & $7(8.1)$ & 0.006 \\
\hline Regorafenib & $5(4.3)$ & $1(1.2)$ & 0.371 \\
\hline Intra-arterial infusion & $5(4.3)$ & $6(7.0)$ & 0.619 \\
\hline
\end{tabular}

Data are shown as numbers of events with percentages in parentheses. TACE, transarterial chemoembolization.

treatments (Table 5). Among all treatments, the rate of receiving ablative therapies (radiofrequency ablation and microwave ablation) was higher in the TACE-apatinib group than in the TACE-sorafenib group. No significant differences were found in other types of subsequent treatments.

\section{Discussion}

The potential of combining targeted agents with TACE in patients with unresectable HCC has been arousing researchers' interests. In this multicenter study, we retrospectively compared the efficacy and safety of TACEapatinib and TACE-sorafenib. Our findings showed that TACE-sorafenib was superior to TACE-apatinib in prolonging the median PFS, but this superiority was not observed in OS. In a subgroup analysis, TACE-sorafenib also demonstrated a superior survival benefit in terms of PFS as compared to TACE-apatinib in the reduced-dose subgroup. However, there were no significant differences in OS and PFS between the two treatment arms in the normal-dose subgroup.

In recent studies, vascular endothelial growth factor has been considered as one of the most important factors associated with hypoxia-induced angiogenesis after TACE (28). However, other cytokines, such as interleukin-6 and tumor necrosis factor- $\alpha$ could also increase after TACE, leading to neo-angiogenesis and tumor growth (29). Distinct from apatinib, sorafenib can inhibit multiple factors involved in the tumor angiogenesis (30). Therefore, the advantage of apatinib in the selectivity of VEGFR-2 might not translate into the superiority of anti-tumor efficacy.

Despite the observed difference in PFS, no significant difference in OS between the two groups was found. We postulate that subsequent treatments after progression could have had some impactful role. As shown in Table 5, a higher proportion of patients received ablative therapies in the TACE-apatinib group than in the TACE-sorafenib group. Generally, ablation is one of the curative therapies for early/very early-stage patients, not recommended for palliative intent (4). However, several studies have reported that debulking intrahepatic tumor burden could improve the prognosis of advanced HCC patients (31-35). Therefore, it is reasonable to hypothesize that more patients receiving ablation in the TACE-apatinib group could have compensated for the inferiority in PFS compared with the TACE-sorafenib group.

Further, we found that neither macrovascular invasion nor distant metastasis was a risk factor of OS, consistent with previous studies (31-33). Unexpectedly, age was a positive prognostic factor of OS and PFS in the entire cohort. In the normal and reduced dose subgroups, elder patients ( $\geq 52$ years old) also had longer OS. Apart from selection bias, we hypothesize that HCC could behave more aggressively and lead to a poorer prognosis in younger patients. Most importantly, TACE-apatinib treatment and dose reduction were confirmed to be independent risk factors for PFS among all patients. Particularly, the patients in the reduced-dose subgroup obtained a more favorable PFS benefit from the TACE-sorafenib than the patients in the entire cohort (reduced subgroup vs. entire cohort: HR $=3.07 v s .1 .53$ ), indicating that the superiority of TACE- 
sorafenib over TACE-apatinib in terms of PFS among all patients might be partly from dose reduction. Thus, dose reduction could attenuate the efficacy of apatinib, and it was attributable to the targeted agent-related AEs in this study.

The incidence of the agent-related AEs in this study is generally consistent with published articles $(6,36,37)$. Here, severe AEs occurred more frequently in the TACE-apatinib group than in the TACE-sorafenib group. This inferior tolerability profile of apatinib might limit its use in clinical practice. If patients cannot tolerate the $\mathrm{AEs}$ of apatinib, a better suggestion might be switching to sorafenib rather than reducing the dose and sequential use of these targeted agents might still prolong the patients' survival. On the other hand, those who can tolerate the AEs and maintain the initial dose of apatinib might obtain equivalent survival benefits to that obtained from sorafenib. At present, the monthly cost of apatinib in China is nearly $30 \%$ lower than that of sorafenib. Therefore, it might become an alternative for those who cannot afford sorafenib. In order to reduce the AEs, some researchers suggest prescribing 500 and $250 \mathrm{mg}$ of apatinib alternatively (37), but further studies are warranted. Besides, lenvatinib, a new targeted agent, was proved to be non-inferior to sorafenib in terms of OS in a phase III HCC trial (38). Considering the high object response rate of lenvatinib (39), comparing the efficacy and safety of TACE-lenvatinib and TACE-sorafenib would be the next stage of our study.

There were several limitations in this study worth mentioning. First, the retrospective nature of this study made it difficult to avoid recall bias. Second, the relatively small sample size of patients in each group and subgroup hindered further stratified analyses. Third, most patients in this study were hepatitis B virus (HBV)-infected (89.1\%) and whether our conclusions could be extended to a non$\mathrm{HBV}$ predominant population also needs verification. Finally, a previous study considers that taking sorafenib prior to first TACE could contribute to the normalization of tumor vessels and improve the efficacy of the combination therapy (11). In this present study, the targeted agents were administrated orally on a non-specific date after the first TACE, which might not reveal the maximal efficacy of the combination therapy.

\section{Conclusions}

TACE-apatinib achieved shorter PFS than TACEsorafenib in advanced HCC patients. However, two treatments exhibited comparable OS. Dose reduction was an independent risk factor associated with PFS and might particularly attenuate the efficacy of apatinib. The safety profile of TACE-apatinib was less well-tolerable than that of TACE-sorafenib. Therefore, TACE-sorafenib could be preferable in the clinical management of advanced HCC but TACE-apatinib could be recommended for the HCC patients who could tolerate the apatinib-associated AEs and maintain the initial dose. All these findings need validation in prospective studies.

\section{Acknowledgments}

We also thank Ms Qiwen Xie, Dr. Suyi Zhang, and Dr. Qi Zhong for polishing our manuscript.

Funding: This work was supported by grants from the National Natural Science Foundation of China (No. 81772598 and 81772625); the Guangdong Provincial Natural Science Foundation of China (No. 2017A030311006); and the Guangzhou Science and Technology Program of China (No. 201804020093).

\section{Footnote}

Reporting Checklist: The authors have completed the STROBE reporting checklist. Available at http://dx.doi. org/10.21037/atm-20-5360

Data Sharing Statement: Available at http://dx.doi. org/10.21037/atm-20-5360

Conflicts of Interest: All authors have completed the ICMJE uniform disclosure form (available at http://dx.doi. org/10.21037/atm-20-5360). The authors have no conflicts of interest to declare.

Ethical Statement: The authors are accountable for all aspects of the work in ensuring that questions related to the accuracy or integrity of any part of the work are appropriately investigated and resolved. In this study, the protocol was approved by the research institutes' committee (No. B2019-153) and was conducted in accordance with the ethical principles of the Declaration of Helsinki (as revised in 2013). Written informed consent was not required due to the retrospective nature of this study.

Open Access Statement: This is an Open Access article distributed in accordance with the Creative Commons Attribution-NonCommercial-NoDerivs 4.0 International 
License (CC BY-NC-ND 4.0), which permits the noncommercial replication and distribution of the article with the strict proviso that no changes or edits are made and the original work is properly cited (including links to both the formal publication through the relevant DOI and the license). See: https://creativecommons.org/licenses/by-nc-nd/4.0/.

\section{References}

1. Siegel RL, Miller KD, Jemal A. Cancer statistics, 2019. CA Cancer J Clin 2019;69:7-34.

2. Feng RM, Zong YN, Cao SM, et al. Current cancer situation in China: good or bad news from the 2018 Global Cancer Statistics? Cancer Commun (Lond) 2019;39:22-33.

3. Forner A, Llovet JM, Bruix J. Hepatocellular carcinoma. Lancet 2012;379:1245-55.

4. European Association for the Study of the Liver. Electronic address: easloffice@easloffice.eu; European Association for the Study of the Liver. EASL Clinical Practice Guidelines: Management of hepatocellular carcinoma. J Hepatol 2018;69:182-236. Erratum in: J Hepatol. 2019 Apr;70(4):817. doi: 10.1016/ j.jhep.2019.01.020. Epub 2019 Feb 7.

5. Llovet JM, Ricci S, Mazzaferro V, et al. Sorafenib in advanced hepatocellular carcinoma. N Engl J Med 2008;359:378-90.

6. Cheng AL, Kang YK, Chen Z, et al. Efficacy and safety of sorafenib in patients in the Asia-Pacific region with advanced hepatocellular carcinoma: a phase III randomised, double-blind, placebo-controlled trial. Lancet Oncol 2009;10:25-34.

7. Sergio A, Cristofori C, Cardin R, et al. Transcatheter arterial chemoembolization (TACE) in hepatocellular carcinoma (HCC): the role of angiogenesis and invasiveness. Am J Gastroenterol 2008;103:914-21.

8. Shim JH, Park JW, Kim JH, et al. Association between increment of serum VEGF level and prognosis after transcatheter arterial chemoembolization in hepatocellular carcinoma patients. Cancer Sci 2008;99:2037-44.

9. Choi GH, Shim JH, Kim MJ, et al. Sorafenib alone versus sorafenib combined with transarterial chemoembolization for advanced-stage hepatocellular carcinoma: results of propensity score analyses. Radiology 2013;269:603-11.

10. Chao Y, Chung YH, Han G, et al. The combination of transcatheter arterial chemoembolization and sorafenib is well tolerated and effective in Asian patients with hepatocellular carcinoma: final results of the START trial. Int J Cancer 2015;136:1458-67.
11. Kudo M, Ueshima K, Ikeda M, et al. Randomised, multicentre prospective trial of transarterial chemoembolisation (TACE) plus sorafenib as compared with TACE alone in patients with hepatocellular carcinoma: TACTICS trial. Gut 2020;69:1492-501.

12. Facciorusso A, Serviddio G, Muscatiello N. Transarterial radioembolization vs chemoembolization for hepatocarcinoma patients: A systematic review and metaanalysis. World J Hepatol 2016;8:770-8.

13. Facciorusso A. Drug-eluting beads transarterial chemoembolization for hepatocellular carcinoma: Current state of the art. World J Gastroenterol 2018;24:161-9.

14. Chu HH, Kim JH, Shim JH, et al. Chemoembolization Plus Radiotherapy Versus Chemoembolization Plus Sorafenib for the Treatment of Hepatocellular Carcinoma Invading the Portal Vein: A Propensity Score Matching Analysis. Cancers (Basel) 2020;12:1116-28.

15. Wang Z, Wang E, Bai W, et al. Exploratory Analysis to Identify Candidates Benefitting from Combination Therapy of Transarterial Chemoembolization and Sorafenib for First-Line Treatment of Unresectable Hepatocellular Carcinoma: A Multicenter Retrospective Observational Study. Liver Cancer 2020;9:308-25.

16. Zhang Y, Miao H, Xie W, et al. The PPRD score stratifies patients with hepatocellular carcinoma and portal vein tumor thrombus treated with sorafenib plus transarterial chemoembolization. Eur Radiol 2021;31:232-43.

17. Tian S, Quan H, Xie C, et al. YN968D1 is a novel and selective inhibitor of vascular endothelial growth factor receptor-2 tyrosine kinase with potent activity in vitro and in vivo. Cancer Sci 2011;102:1374-80.

18. Qin SK. Apatinib in Chinese patients with advanced hepatocellular carcinoma: a phase II randomized, openlabel trial. J Clin Oncol 2014;32:abstract 4019.

19. Lu W, Jin XL, Yang C, et al. Comparison of efficacy between TACE combined with apatinib and TACE alone in the treatment of intermediate and advanced hepatocellular carcinoma: A single-center randomized controlled trial. Cancer Biol Ther 2017;18:433-8.

20. Chen S, Yu W, Zhang K, et al. Comparison of the efficacy and safety of Transarterial chemoembolization with and without Apatinib for the treatment of BCLC stage C hepatocellular carcinoma. BMC Cancer 2018;18:1131-8.

21. Fan W, Yuan G, Fan H, et al. Apatinib Combined with Transarterial Chemoembolization in Patients With Hepatocellular Carcinoma and Portal Vein Tumor Thrombus: A Multicenter Retrospective Study. Clin Ther 2019;41:1463-76. 
22. Qiu Z, Shen L, Chen S, et al. Efficacy of Apatinib In Transcatheter Arterial Chemoembolization (TACE) Refractory Intermediate And Advanced-Stage Hepatocellular carcinoma: A Propensity Score Matching Analysis. Cancer Manag Res 2019;11:9321-30.

23. Yang Z, Chen G, Cui Y, et al. The safety and efficacy of TACE combined with apatinib on patients with advanced hepatocellular carcinoma: a retrospective study. Cancer Biol Ther 2019;20:321-7.

24. Zhao S, Zhang T, Dou W, et al. A comparison of transcatheter arterial chemoembolization used with and without apatinib for intermediate- to advanced-stage hepatocellular carcinoma: a systematic review and metaanalysis. Ann Transl Med 2020;8:542.

25. Peng Z, Chen S, Xiao H, et al. Microvascular Invasion as a Predictor of Response to Treatment with Sorafenib and Transarterial Chemoembolization for Recurrent Intermediate-Stage Hepatocellular Carcinoma. Radiology 2019;292:237-47.

26. Lencioni R, Llovet JM. Modified RECIST (mRECIST) assessment for hepatocellular carcinoma. Semin Liver Dis 2010;30:52-60.

27. Cheng AL, Kang YK, Lin DY, et al. Sunitinib versus sorafenib in advanced hepatocellular cancer: results of a randomized phase III trial. J Clin Oncol 2013;31:4067-75.

28. Petrillo M, Patella F, Pesapane F, et al. Hypoxia and tumor angiogenesis in the era of hepatocellular carcinoma transarterial loco-regional treatments. Future Oncol 2018;14:2957-67.

29. Chao Y, Wu CY, Kuo CY, et al. Cytokines are associated with postembolization fever and survival in hepatocellular carcinoma patients receiving transcatheter arterial chemoembolization. Hepatol Int 2013;7:883-92.

30. Wilhelm SM, Carter C, Tang L, et al. BAY 43-9006 exhibits broad spectrum oral antitumor activity and targets the RAF/MEK/ERK pathway and receptor tyrosine kinases involved in tumor progression and angiogenesis. Cancer Res 2004;64:7099-109.

31. Okusaka T, Okada S, Ishii H, et al. Prognosis of

Cite this article as: Qiu Z, Shen L, Jiang Y, Qiu J, Xu Z, Shi M, Yu Z, Ma Y, He W, Zheng Y, Li B, Wang G, Yuan Y. Transarterial chemoembolization (TACE) combined with apatinib versus TACE combined with sorafenib in advanced hepatocellular carcinoma patients: a multicenter retrospective study. Ann Transl Med 2021;9(4):283. doi: 10.21037/atm-205360 hepatocellular carcinoma patients with extrahepatic metastases. Hepatogastroenterology 1997;44:251-7.

32. Uka K, Aikata H, Takaki S, et al. Clinical features and prognosis of patients with extrahepatic metastases from hepatocellular carcinoma. World J Gastroenterol 2007;13:414-20.

33. Yoo DJ, Kim KM, Jin YJ, et al. Clinical outcome of 251 patients with extrahepatic metastasis at initial diagnosis of hepatocellular carcinoma: does transarterial chemoembolization improve survival in these patients? J Gastroenterol Hepatol 2011;26:145-54.

34. Tanaka K, Yabushita Y, Nakagawa K, et al. Debulking surgery followed by intraarterial 5-fluorouracil chemotherapy plus subcutaneous interferon alfa for massive hepatocellular carcinoma with multiple intrahepatic metastases: a pilot study. Eur J Surg Oncol 2013;39:1364-70.

35. de Stefano G, Farella N, Scognamiglio U, et al. Sorafenib after RFA in HCC patients: a pilot study. Hepatogastroenterology 2015;62:261-3.

36. Li J, Qin S, Xu J, et al. Apatinib for chemotherapyrefractory advanced metastatic gastric cancer: results from a randomized, placebo-controlled, parallel-arm, phase II trial. J Clin Oncol 2013;31:3219-25.

37. Lan CY, Wang Y, Xiong Y, et al. Apatinib combined with oral etoposide in patients with platinum-resistant or platinum-refractory ovarian cancer (AEROC): a phase 2, single-arm, prospective study. Lancet Oncol 2018;19:1239-46.

38. Kudo M, Finn RS, Qin S, et al. Lenvatinib versus sorafenib in first-line treatment of patients with unresectable hepatocellular carcinoma: a randomised phase 3 noninferiority trial. Lancet 2018;391:1163-73.

39. Matsui J, Yamamoto Y, Funahashi Y, et al. E7080, a novel inhibitor that targets multiple kinases, has potent antitumor activities against stem cell factor producing human small cell lung cancer H146, based on angiogenesis inhibition. Int J Cancer 2008;122:664-71. 